\title{
System for generating dynamic random-element stereograms
}

\author{
SUDHAKAR S. SHETTY, ARTHUR J. BRODERSEN, and ROBERT FOX \\ Vanderbilt University, Nashville, Tennessee 37240
}

\begin{abstract}
Truly dynamic stereograms can be generated by a new system that produces an anaglyphic display on a commercial television receiver. Location, size, and disparity (both direction and magnitude) of the stereographic form are controlled by means of a TTL unit.
\end{abstract}

Random-element stereograms and their extensive application to both applied and basic lines of inquiry are well known (e.g., Julesz, 1971). The most widely used technique for stereogram construction involves generating a matrix of achromatic dots (matrix size usually 100 by 100 ) on a computer-controlled graphic display and then making a hard copy (i.e., a photograph) of the displayed matrix. These stereograms are referred to as random-element because the positions of elements within the matrix have been specified on a random basis; yet, in other respects, the stereograms are static in that all attributes of the embedded stereoscopic form-shape, disparity value, and so on-cannot be changed. But for many applications, it is highly desirable, if not essential, to use truly dynamic stereograms in which stereoscopic forms appear to move continuously about in stereoscopic space. An early method for producing truly dynamic stereograms used cinematographic techniques. A number of films showing stereographic forms in motion have been made (see Julesz, 1971). But film is a cumbersome and expensive method that still imposes many restrictions on the way stereoscopic contours can be manipulated.

Recently, advances in microcomputer and integratedcircuit technology have made it possible to generate dynamic dot matrices directly on CRT displays. The left-eye and right-eye matrices are presented either on separate CRTs or spatially separated on a single CRT and then brought into binocular coincidence by optical devices such as haploscopes and prisms. Stereogram generation systems of this type have been described by Bouldin (1975), Julesz, Breitmeyer, and Kropfl (1976), Ross and Hogben (1974), Uttal, Fitzgerald, and Eskin (1975), and Miller (Note 1). Typically, these systems require both a minicomputer and an external hard-wired special-purpose electronic device. The external device is used for high-speed repetitive tasks such as the continuous generation of random dots. The minicomputer

This work was supported by Grant EY00590 from the National Institutes of Health and by Contract 197-036 from the Office of Naval Research. For their comments on an earlier version of the manuscript, we wish to thank Robert H. Cormack and Randolph Blake. Requests for reprints should be sent to Robert Fox, Vanderbilt University, Department of Psychology, Nashville, Tennessee 37240. keeps track of the displayed positions of the dots and inserts disparity at those points in the $\mathrm{X}$-Y coordinate space of the display that are specified by the program. Although these systems generate truly dynamic stereograms, they have several limitations: (1) The use of a minicomputer restricts portability and makes the system relatively expensive. (2) Alignment of matrices for dichoptic stimulation requires the active cooperation of the observer. (Note also that haploscopic viewing systems can disrupt normal accommodation and convergence relationships and thus may impair the perception of stereopsis.) (3) Matrix size and dot density are often reduced by the restrictions imposed by computer cycle time. (4) The luminance of the dot matrices is inherently low. (5) Changes in display parameters can require changes in software, which may be formidable.

We now describe a system that overcomes these limitations by capitalizing upon even more recent advances in microelectronic technology. The system can use as a display almost any type of commercial color television receiver. Large matrices of red and green dots are continuously generated, and the dichoptic stimulation requisite for stereopsis is produced by placing appropriate chromatic filters before the eyes of the observer. The filters physically block wavelengths so that only one matrix, either red or green, can stimulate a single eye. This is, of course, the well known anaglyph method for stereoscopic presentation (Woodworth, 1938). The system for generating and controlling the display consists of a hard-wired network of approximately 60 7400-series TTL chips housed in a selfcontained unit. Parameters of the stereoscopic form that can be quickly varied include disparity magnitude and direction, $\mathrm{X}-\mathrm{Y}$ locus, and rectilinear configuration. Several versions of the system have been successfully used in investigations of stereopsis in both animals and humans (Fox, Lehmkuhle, \& Bush, 1977; Fox, Lehmkuhle, \& Leguire, 1978; Lehmkuhle \& Fox, Note 2).

\section{OVERVIEW OF THE SYSTEM}

The red and green dot matrices are produced on the color video display by modulating the inputs to the red and green electron guns as they sweep the display in the 
raster-scan mode. All dots comprising the matrices can be randomly replaced at the completion of every frame by means of a randon-number generator. Delaying the onset of one gun at some point during the scan produces a spatial difference in the X-Y positions of the red and green dots; this difference in the location of the dots is the sine qua non of the retinal disparity required for stereopsis. The spatial difference produced by the time delay is camouflaged by adding random dots that are not correlated with the left-eye and red-eye dot matrices. By maintaining control of the X-Y positions of the guns as they sweep the display, disparity and the correlative stereoscopic form can be introduced at any position within the display. Figure 1 illustrates the main components of the electronic unit that governs the display.

The electronic unit connects with the display at the level of the output amplifiers, and synch signals are injected at the level of the synch separator. These inputs require only modest modifications of a color video receiver and do not permanently alter the receiver's normal functions. In the circuit, shown in Figure 2, the $X_{1}$ input would be from the green color amplifier of Figure 1, and the video input would be grounded. An identical connection would be made for the red color amplifier. The specific method for interfacing with a commercial television receiver varies with the manufacturer and model. (See Lancaster, 1976, for a practical discussion of methods.)

\section{SWEEP GENERATOR}

A functional schematic of the sweep generator is shown in Figure 3. The generator divides the $.5 \cdot \mathrm{mHz}$ input from the clock into vertical and horizontal synchronization pulses. The entire circuit consists of three

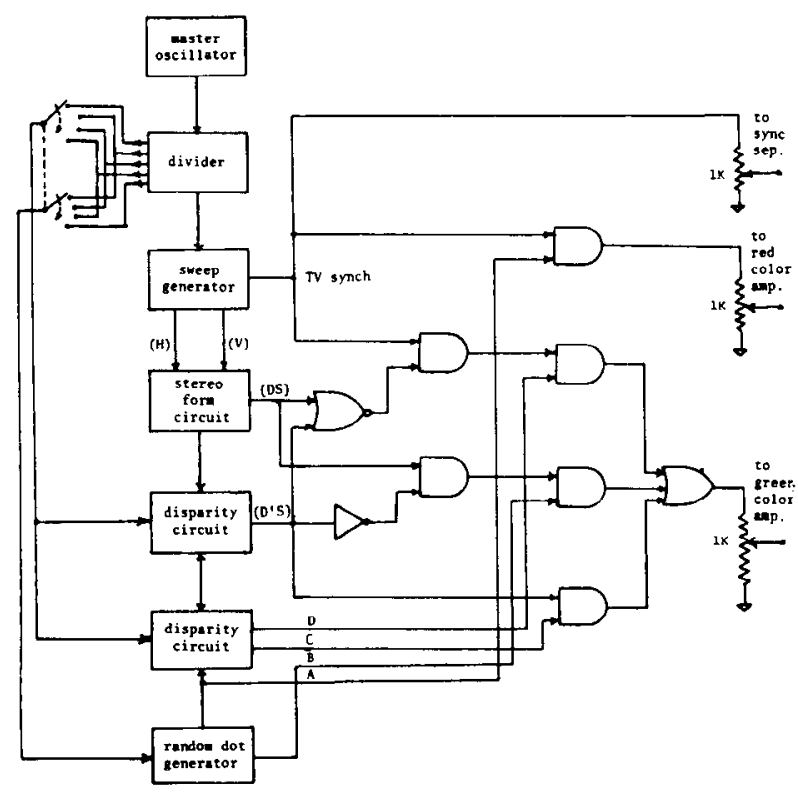

Figure 1. Block diagram of stereogram generator.

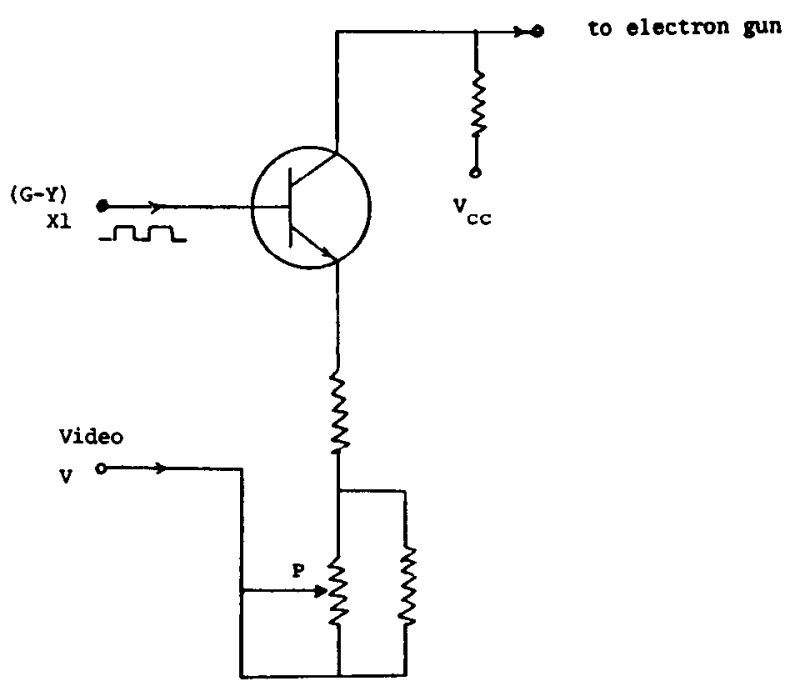

Figure 2. Typical output of television color amplifier.

dual-D flip-flops (7474), two 4-bit binary counters (7493), two quadruple NAND gates (7400), one quadruple NOR gate (7402), and one hex converter (7404). The flip-flop outputs, in conjunction with the NAND and NOR gates, generate the horizontal output, which has a period of 54 microsec and a retrace time of 10 microsec. Note that the actual horizontal frequency of a commercial television is 53.5 microsec, in contrast to the 54 microsec produced by this circuit, but this difference does not affect the performance of the system. The binary counters and the NAND and NOR gates are used to generate the timing pulses for the vertical output. The slight deviation of this vertical period $(16.4 \mathrm{msec})$ from that of a commercial unit does not affect the display.

The vertical and horizontal signals are combined into a single waveform at the NOR gate. The combined signal is then fed to the base of the separator stage of the receiver.

\section{STEREOSCOPIC FORM CIRCUIT}

Figure 4 represents a functional schematic of the stereoscopic form circuit. This circuit designates a portion of the sweep for conversion to stereoscopic presentation. Provision is made for varying the position and the dimensions of the form. The circuit consists of four negative-edge, triggered, monostable multivibrators and a NAND gate. The four variable resistors, $R_{1} \cdot R_{4}$ in Figure 4 , are used to vary the shape, size, and position of the stereoscopic form. $\mathbf{R}_{1}$ and $\mathbf{R}_{2}$ vary the position and the size, respectively, of the form in the $X$ or horizontal dimension. $R_{3}$ and $R_{4}$ vary the position and size, respectively, in the $Y$ or vertical dimension. By adjusting $R_{2}$ and $R_{4}$, any rectilinear shape of any size can be presented. By continuous variation of $R_{1}$ and $R_{3}$, the shape can be made to appear to move continuously in either horizontal or vertical directions. 
clock

$(0.5=\mathrm{Hz})$

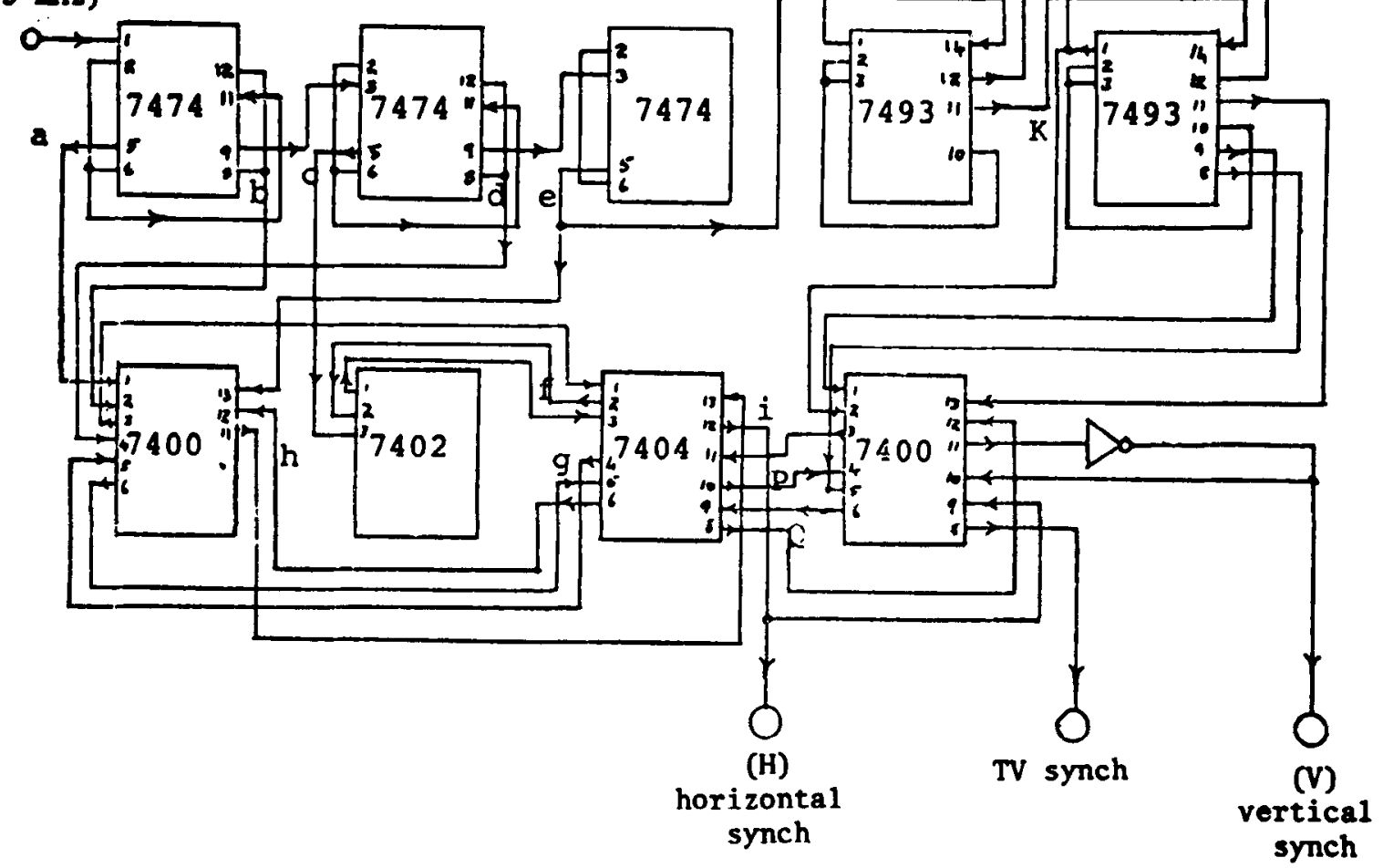

Figure 3. Sweep generator.

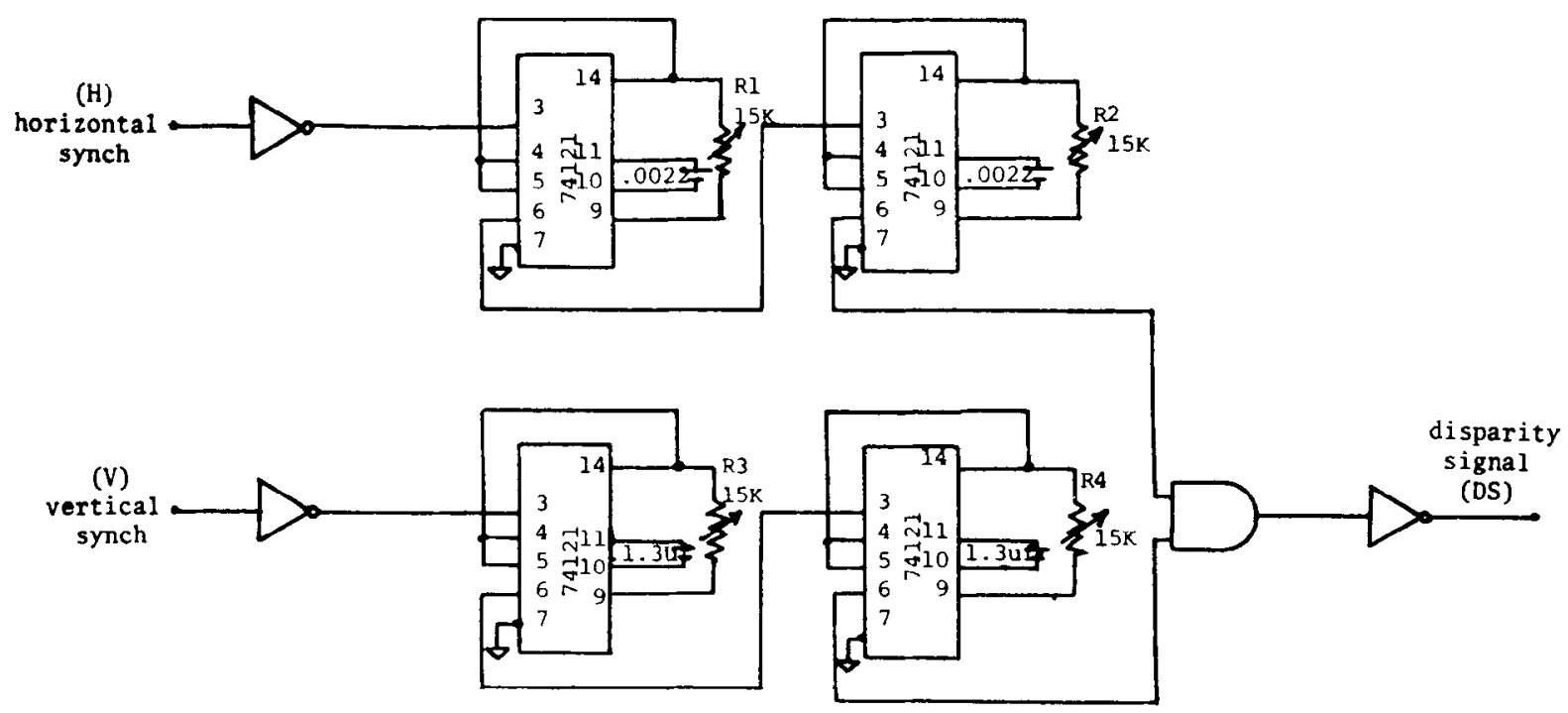

Figure 4. Stereoscopic form circuit.

\section{DISPARITY CIRCUIT}

The system produces retinal disparity by spatially shifting the form, with respect to the background, in one matrix. The random pulses that comprise the form are delayed independently in the red and green matrices but remain in correlation; that is, both are delayed separately by equal amounts and then combined via a NAND gate. The correlation is necessary to keep the red and green matrices aligned.
A complete schematic of the disparity circuit is shown in Figure 5. The heart of the circuit is a set of six 8-bit parallel-out serial shift registers. Three shift registers are used for delay of the stereoscopically presented form, and three others are used for delay of the random pulses. The circuit generates eight delay steps of two dots each, that is, two columns of dots for each delay step. To avoid monocular cues, the delayed matrix is carefully interlaced between the background dots by selecting only odd dot delays. The addressing 


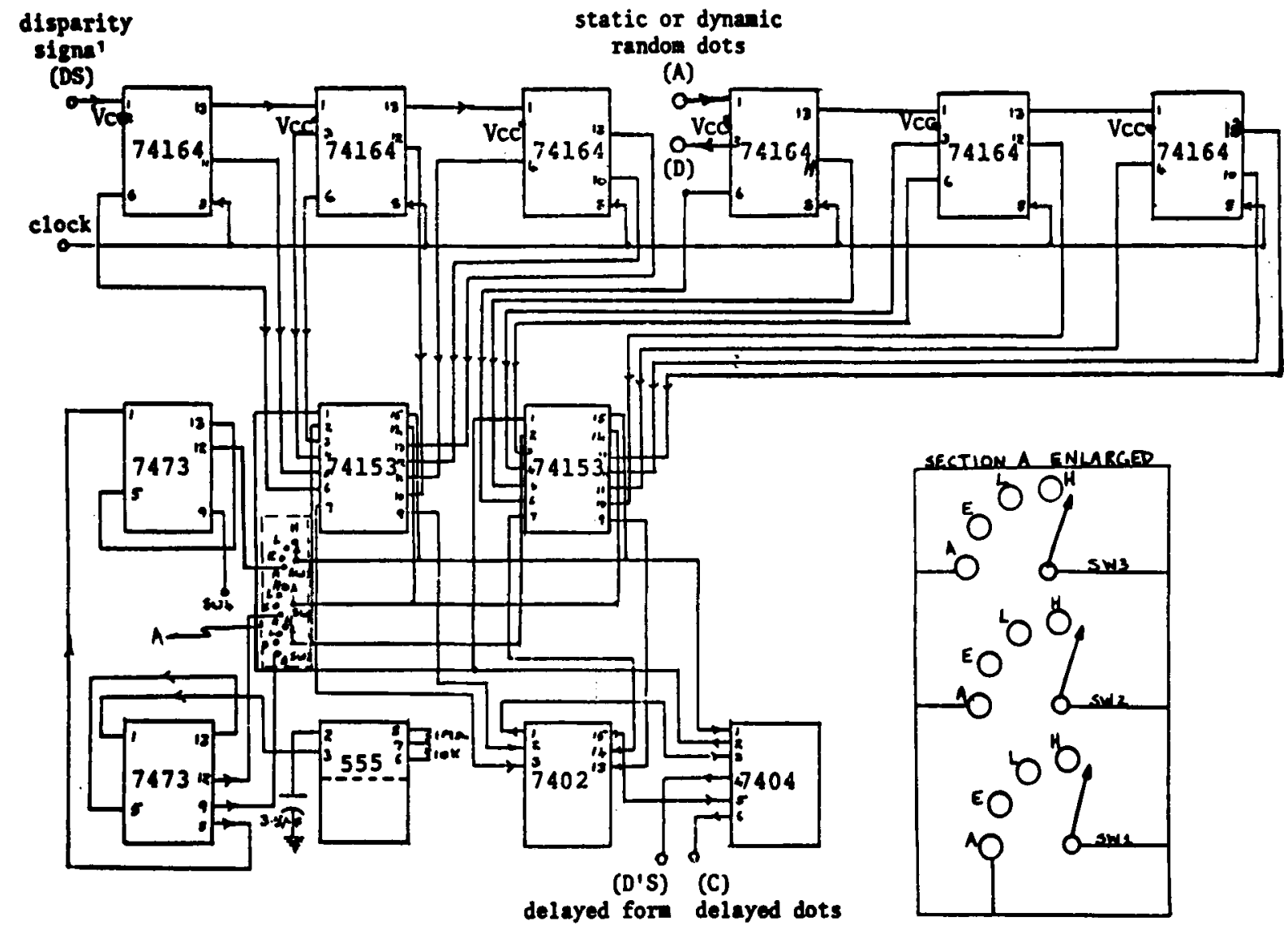

Figure 5. Disparity circuit.

system of these eight delay steps is comprised of two eight-line/one-line data selectors (74150). Provision for automatic delay selection is made by using a 555 timer (two 7473 circuits). The timer controls the shift rate.

\section{RANDOM-DOT GENERATOR}

The display can be presented in two modes, static or dynamic. In the static display, the same set of random dots is repeatedly displayed on every frame, and the resulting display is much like a photograph. In the dynamic display, a different set of random dots is presented on each frame, and the resulting display appears to have a sparkling surface due to the apparent motion of the dots. However, this continual motion is correlated between the red and green matrices, and there is absolutely no interference with the stereoscopic form itself, which remains as sharp and clear as the form presented in the static mode.

A functional schematic of the random-dot generator is shown in Figure 6. The circuit uses a pseudorandom sequence generated by a chain of shift registers. The length of the sequence for an $n$-stage register is $2^{n}-1$. Three shift registers (74164) with an exclusive OR gate produce a sequence of length $2^{24}-1=16,766,977$. In this application, using a $2-\mathrm{mHz}$ clock, the length of the sequence necessary to fill the screen is 33,000 .
Therefore, the sequence of random dots will be repeated every 500 frames or $8,000 \mathrm{msec}$. Since the sequence changes every frame without repetition for 500 frames, the display appears to be truly random. Other dot sequences could be obtained by changing the feedback of the exclusive OR gate from Pins 19 and 24 of the shift register to any of the following: Pins 18 and 23 , 21 and 22,19 and 21 , or 17 and 20 .

To produce a static display in which the same randomdot pattern is presented at every frame, the shift registers are cleared after every vertical blanking. The first-stage output of the shift register is used to fill the small area left blank by the shift. This output, which differs from the 24th-stage output, fills in the gap without producing disparity.

\section{COMMENTS ON THE ANAGLYPH METHOD}

Although the anaglyph method for producing dichoptic stimulation for stereoscopic presentation is certainly not a new technique, some of its characteristics are still not common knowledge. For instance, it is occasionally suggested that the method requires color vision on the part of the observer. This is not correct. The chromatic filters produce image separation by physically blocking wavelength. When an anaglyphic display is viewed without chromatic filters, the spatial separation 


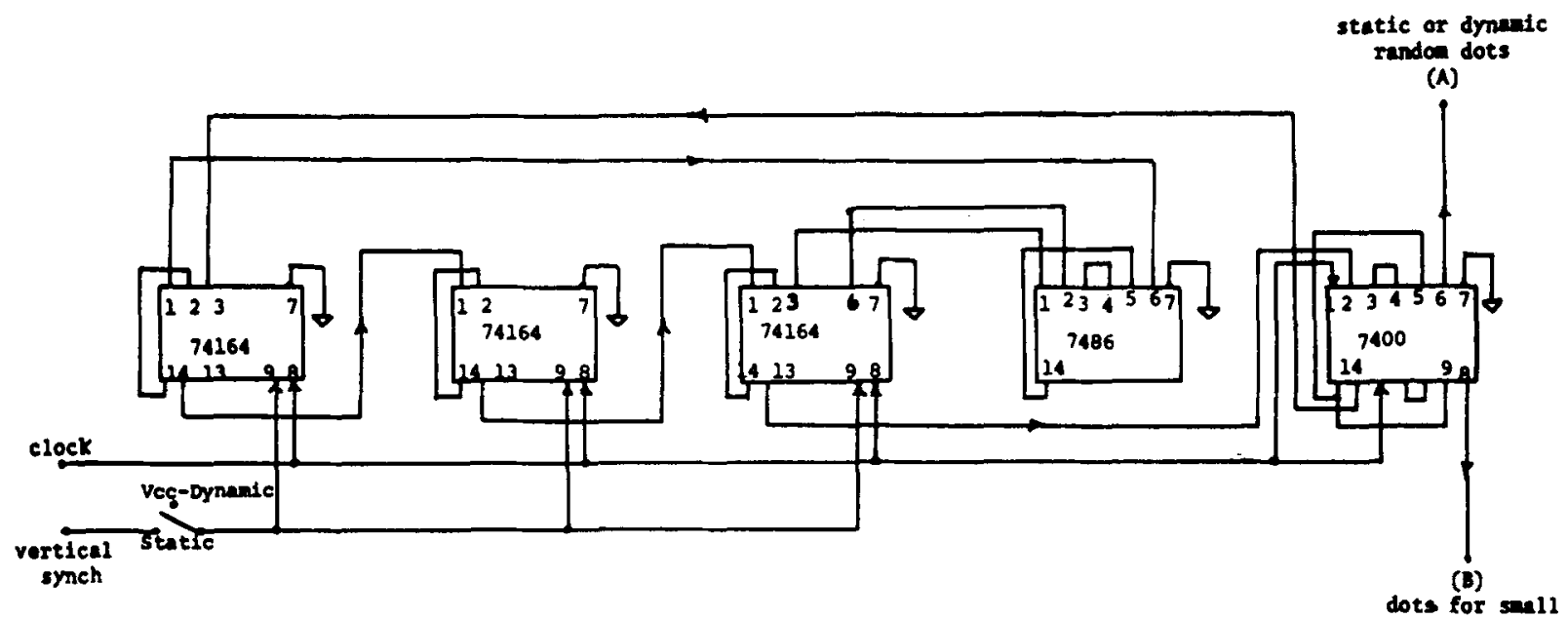

Figure 6. Random dot generator.

between portions of the display containing disparity can be seen. This gives rise to the concern that the separation may give clues about the nature of the stereoscopic form. Such clues, of course, are inevitable whenever the left and right views of any stereogram are optically superimposed. But they are not present when the views are segregated and seen by separate eyes.

With respect to view segregation, it is sometimes suggested that filter methods such as the analygphic and polarization schemes are less effective (i.e., permit more cross talk) than optical methods such as provided by haploscopes. While, in principle, cross talk can be present in almost any filter segregation system, it probably has no practical significance. For instance, in a truly dynamic random-element stereogram, the continual replacement of elements and the absence of binocularly visible contours suppress the presence of useful monocular cues, even when cross talk is considerable.

In applications of the present stereogram generation system, the perceptual consequences of cross talk have proven to be negligible. Using Wratten filters for the eyes of the observers (29 "red," 58 "green"), a variety of television receivers have been used quite successfully. These include Hitachi, Zenith, and RCA commercial solid state 19-in. color television receivers and Advent 1000 and 750 projection-type video receivers. For these applications, the degree of cross talk has been assessed not only by visual inspection but also by psychophysical experiments wherein a sophisticated observer wearing identical filters before both eyes is required to make a forced-choice judgment of the spatial position of a stereoscopic form in X-Y coordinates. Even with feedback after every trial and extended practice, the observers have not been able to make discriminations above the chance level. These kinds of tests are a more conservative and conclusive way of determining the effect of cross talk than efforts to match phosphors and filters in terms of their nominal spectral characteristics.

\section{CONSTRUCTION NOTES}

Construction is straightforward, but, because of high chip density, certain precautions should be taken to minimize capacitance and inductance effects. Components should be placed to minimize connection length. A .01-microF bypass capacitor should be placed across each chip. Separate bus lines should be run for each row of chips. Construction should probably be undertaken only by someone with considerable experience in integrated circuit assembly. Wiring mistakes and chip failure are almost inevitable; hence, the use of wirewrap methods is highly recommended.

The choice of a color television receiver does not seem to be critical from the electronic standpoint. Note that most receivers have the power line connected to the chassis. This raises the possibility of a severe shock hazard. It is essential that both devices remain isolated from an earth ground. Two-prong plugs and/or isolation transformers should be used when connecting to the power line. (For a discussion of shock hazard and methods of construction, see Lancaster, 1976.)

The cost of parts for the stereogram generator should not exceed $\$ 300$. The single most expensive component is the $5 . \mathrm{V}$ power supply $(\$ 30)$. The integrated circuits, transistors, and capacitors are all commercial grade.

\section{REFERENCE NOTES}

1. Miller, D. D. A device for the generation and display of dynamic random-dot stereograms. Paper presented at the meeting of the Association for Research in Vision and Opthalmology, Sarasota, May 1974.

2. Lehmkuhle, S. W., \& Fox, R. Global stereopsis in the cat. Paper presented at the meeting of the Association for Research in Vision and Opthalmology, Sarasota, May 1977. 


\section{REFERENCES}

Bouldin, D. W. Visually evoked cortical potentials elicited by dynamic random-dot stereograms. Unpublished doctoral dissertation, Vanderbilt University, 1975.

Fox, R., Lehmкuhle, S. W., \& Bush, R. C. Stereopsis in the falcon. Science, 1977, 197, 79-81.

Fox, R., Lehmкuhle, S. W., \& Leguire, L. E. Stereoscopic contours induce optokinetic nystagmus. Vision Research, 1978, 18, 1189-1192.

JULESz, B. Foundations of cyclopean perception. Chicago: University of Chicago Press, 1971.

Julesz, B., Breitmeyer, B., \& Kropfl, W. Binocular-disparitydependent upper-lower hemifield anistropy and left-right hemi- field isotropy as revealed by dynamic random-dot stereograms. Perception, 1976, 5, 129-141.

LANCASter, D. TTL typewriter cookbook. Indianapolis: Sams, 1976.

Ross, J., \& Hogben, J. H. Short-term memory in stereopsis. Vision Research, 1974, 14, 1195-1201.

Uttal, W. R., Fitzgerald, J., \& Eskin, T. E. Parameters of tachistoscopic stereopsis. Vision Research, 1975, 15, 705-712.

Woodworth, R. S. Experimental psychology. New York: Holt, 1938.

(Received for publication March 20, 1979; revision accepted July 16, 1979.) 